\title{
On the Practical Stability of Impulsive Differential Equations with Infinite Delay in Terms of Two Measures
}

\author{
Bo Wu, ${ }^{1,2}$ Jing Han, ${ }^{3}$ and Xiushan Cai ${ }^{1}$ \\ ${ }^{1}$ College of Mathematics, Physics and Information Technology, Zhejiang Normal University, \\ Jinhua 321004, China \\ ${ }^{2}$ Academic Affairs Division, Zhejiang Normal University, Jinhua 321004, China \\ ${ }^{3}$ Department of Mathematics, Tongji University, Shanghai 200092, China
}

Correspondence should be addressed to Bo Wu, wubo_502@163.com

Received 13 April 2012; Accepted 18 May 2012

Academic Editor: Chaitan Gupta

Copyright (C) 2012 Bo Wu et al. This is an open access article distributed under the Creative Commons Attribution License, which permits unrestricted use, distribution, and reproduction in any medium, provided the original work is properly cited.

We consider the practical stability of impulsive differential equations with infinite delay in terms of two measures. New stability criteria are established by employing Lyapunov functions and Razumikhin technique. Moreover, an example is given to illustrate the advantage of the obtained result.

\section{Introduction}

One of the trends in the stability theory of the solutions of differential equations is the socalled practical stability, which was introduced by LaSalle and Lefschetz [1]. This is very useful in estimating the worst-case transient and steady-state responses and in verifying pointwise in time constraints imposed on the state trajectories. Fundamental results in this direction were obtained in [2]. In recent years the theory of practical stability and stability has been developed very intensively [3-7].

The theory of impulsive differential equations is now being recognized to be not only richer than the corresponding theory of differential equations without impulses, but also represents a more natural framework for mathematical modelling of many real world phenomena. Impulsive differential equations and impulsive functional differential equations have been intensively researched [8-20].

By employing the Razumikhin technique and Lyapunov functions, several stability criteria are established for general impulsive differential equations with finite delay [5$7,14,21]$. Systems with infinite delay deserve study because they describe a kind of system 
present in the real world. For example, it is very useful in a predator-prey system. Therefore, it is an interesting and complicated problem to study the stability of impulsive functional differential systems with infinite delay. Usually, the Lyapunov functions are defined on whole components of system's state $x$ [12-22]. In this paper, we divided the components of $x$ into several groups and correspondingly, we employ several Lyapunov functions $V_{j}\left(t, x^{(j)}\right)(j=$ $1,2, \ldots, m)$, where $x=\left(x^{(1)}, \ldots, x^{(m)}\right)^{T}$ for each $x^{(j)}$. In this way, Lyapunov, functions are easier constructed, and the conditions ensuring the required stability are less restrictive. Furthermore, the stability results on impulsive finite delay differential equations considered in $[4,5]$ are generalized into the results on impulsive infinite delay differential equations in terms of two measures.

The work is organized as follows. In Section 2, we introduce some preliminary definitions which will be employed throughout the paper. In Section 3, based on Lyapunov functions and Razumikhin method, sufficient conditions for the uniformly practical stability in terms of two measures are given; an example is presented to illustrate the effectiveness of the approach.

\section{Preliminaries}

Consider the following impulsive infinite delay differential equations:

$$
\begin{gathered}
\dot{x}(t)=f(t, x(s) ; \alpha \leq s \leq t), \quad t \geq t_{*}, \quad t \neq \tau_{k}, \\
\Delta x(t) \triangleq x(t)-x\left(t^{-}\right)=I_{k}\left(x\left(t^{-}\right)\right), \quad t=\tau_{k}, k=1,2, \ldots,
\end{gathered}
$$

where $-\infty \leq \alpha<t_{*}, \alpha$ could be $-\infty, t \in R^{+}, f \in C\left[R^{+} \times P C\left([\alpha, t], R^{n}\right), R^{n}\right]$ is a Volterratype function. $P C\left([\alpha, t], R^{n}\right)$ denotes the space of piecewise right continuous functions $\varphi=$ $\left(\varphi_{1}, \ldots, \varphi_{n}\right):[\alpha, t] \rightarrow R^{n}$ with the sup-norm $\|\varphi\|=\sup _{\alpha \leq s \leq t}|\varphi(s)|, \quad|\varphi(s)|=\max _{1 \leq j \leq n}\left|\varphi_{j}(s)\right|$, $f(t, 0) \equiv 0, I_{k}(0)=0,0=\tau_{0}<\tau_{1}<\tau_{2}<\cdots<\tau_{k}<\cdots, \tau_{k} \rightarrow \infty$ for $k \rightarrow \infty$, and $x\left(t^{-}\right)=\lim _{s \rightarrow t^{-}} x(s)$. The functions $I_{k}: R^{n} \rightarrow R^{n}, k=1,2, \ldots$, are such that if $\|x\|<H$ and $I_{k}(x) \neq 0$, then $\left\|x+I_{k}(x)\right\|<H$, where $H=$ const. $>0$.

The initial condition for system (2.1) is given by

$$
x(t)=\varphi(t), \quad t \in\left[\alpha, t_{0}\right],
$$

where $\varphi \in P C\left(\left[\alpha, t_{0}\right], R^{n}\right)$, for $t_{0} \geq t_{*}$.

We assume that a solution for the initial problem (2.1) and (2.2) does exist and is unique. Since $f(t, 0)=0$, then $x(t)=0$ is a solution of $(2.1)$, which is called the zero solution. Let $P C_{\rho}(t)=\left\{\varphi \in P C\left([\alpha, t], R^{n}\right) \mid\|\varphi\|<\rho\right\}$. For convenience, we define $|x|:=$ $\max _{1 \leq i \leq n}\left|x_{i}\right|, x \in R^{n} ; R_{\alpha}:=[\alpha, \infty) ; S(\rho)=\left\{x \in R^{n}:\|x\|<\rho\right\} ; S^{(j)}(\rho)=\left\{x \in R^{n_{j}} \mid\|x\|<\right.$ $\rho\}, K:=\left\{W \in C\left[R^{+}, R^{+}\right], W(0)=0 ; W(s)>0, s>0\right\}, \Gamma^{n}:=\left\{h \in C\left[R^{+} \times R^{n}, R^{+}\right] \mid \forall t \in\right.$ $\left.R^{+}, \inf _{x} h(t, x)=0\right\}, \Gamma_{\alpha}^{n}:=\left\{h \in C\left[R_{\alpha} \times R^{n}, R^{+}\right] \mid \forall t \in R_{\alpha}, \inf _{x} h(t, x)=0\right\}$.

Definition 2.1. A continuous function $w: R^{+} \rightarrow R^{+}$is called a wedge function if $w(0)=0$ and $w(s)$ is (strictly) increasing. 
Definition 2.2. For $h_{0} \in \Gamma_{\alpha}^{n}, x_{t}(s):=x(s), s \in[\alpha, t]$ and $x_{t} \in P C\left\{[\alpha, t], R^{n}\right\}$, for any $t \in R^{+}$, we define

$$
\tilde{h}_{0}\left(t, x_{t}\right)=\sup _{\alpha \leq \theta \leq t} h_{0}(\theta, x(\theta))
$$

Definition 2.3 (see[22]). Let $h_{0} \in \Gamma_{\alpha}^{n}, h \in \Gamma^{n}$. The impulsive functional differential 1 (2.1), (2.2) is said to be

(S1) $\left(\tilde{h}_{0}, h\right)$ practically stable, if given $(u, v)$ with $0<u<v$, we have $\widetilde{h}_{0}\left(t_{0}, x_{t_{0}}\right)<u$ implies $h(t, x)<v, t \geq t_{0}$ for some $t_{0} \in R^{+}$;

(S2) $\left(\tilde{h}_{0}, h\right)$ uniformly practically stable if (S1) holds for every $t_{0} \in R^{+}$.

In what follows, we will split $\varphi=\in P C(\rho)$ into several vectors, such that $\sum_{i=1}^{m} n_{i}=$ $n$ and $\varphi=\left(\varphi_{1}^{(1)}, \ldots, \varphi_{n_{1}}^{(1)}, \varphi_{1}^{(2)}, \ldots, \varphi_{n_{2}}^{(2)}, \ldots, \varphi_{1}^{(m)}, \ldots, \varphi_{n_{m}}^{(m)}\right)^{T}$. For convenience, we define $\varphi^{(j)}=$ $\left(\varphi_{1}^{(j)}, \varphi_{2}^{(j)}, \ldots, \varphi_{n_{j}}^{(j)}\right), j=1,2, \ldots, m$, and $\varphi=\left(\varphi^{(1)}, \varphi^{(2)}, \ldots, \varphi^{(m)}\right)^{T}$. For $x=\left(x_{1}, x_{2}, \ldots, x_{n}\right)^{T} \in$ $R^{n}$, we adopt notation as for $\varphi$. Similarly, let $\left\|\varphi^{(j)}\right\|=\left\|\varphi^{(j)}\right\|^{[\alpha, t]}=\sup _{\alpha \leq s \leq t}\left|\varphi^{(j)}\right|, P C^{(j)}(t)=$ $\left\{\varphi^{(j)}:[\alpha, t] \rightarrow R^{n_{j}} \mid \varphi^{(j)}\right.$ is piecewise continuous and bounded $\}$, and $S^{(j)}(\rho)=\left\{x \in R^{n_{j}} \mid\right.$ $\|x\|<\rho\}, P C_{\rho}^{(j)}(t)=\left\{\varphi^{(j)} \in P C^{(j)}(t) \mid\left\|\varphi^{(j)}\right\|<\rho\right\}$.

\section{Main Results}

In the sequence, we assume that $f$ is defined on $R_{\alpha} \times P C_{H}(t)$ for some $H>0$. For simplicity, denote $V_{i}\left(t, x^{(i)}\right), h^{(i)}\left(t, x^{(i)}\right), h_{0}^{(i)}\left(t, x^{(i)}\right)$ by $V_{i}(t), h^{(i)}(t), h_{0}^{(i)}(t)$, respectively, $1 \leq i \leq m$. Now we start with the case of $m=2$. $V^{\prime}(t)$ be the right-hand derivative of $V(t)$.

Theorem 3.1. For $j=1,2$, let $\Phi_{j}: R^{+} \rightarrow R^{+}$be continuous, $\Phi_{j} \in L^{1}[0, \infty), \Phi_{j}(t) \leq K_{j}$ for $t \geq 0$ with some constants $K_{j}>0$, and let $W_{i j}(i=1,2,3,4)$ be wedge functions. If there exist Lyapunov functions $V_{j}: R_{\alpha} \times S^{(j)}(H) \rightarrow R^{+}(j=1,2)$ such that

(i) $W_{1 j}\left(h^{(j)}(t)\right) \leq V_{j}(t) \leq W_{2 j}\left(h_{0}^{(j)}(t)\right)+W_{3 j}\left[\int_{\alpha}^{t} \Phi_{j}(t-s) W_{4 j}\left(h_{0}^{(j)}(t)\right) d s\right]$, where $h_{0}^{(j)} \in$ $\Gamma_{\alpha}^{n_{j}}, h^{(j)} \in \Gamma^{n_{j}} ;$

(ii) when $V_{1}(t) \geq V_{2}(t)$, there holds $V_{1}^{\prime}(t) \leq 0$ if $V_{1}(s)<V_{1}(t)$ for $s \in[\alpha, t]$; when $V_{2}(t) \geq$ $V_{1}(t)$, there holds $V_{2}^{\prime}(t) \leq 0$ if $V_{2}(s)<V_{2}(t)$ for $s \in[\alpha, t]$;

(iii) $V_{j}\left(\tau_{k}\right) \leq\left(1+b_{k}\right) V_{j}\left(\tau_{k}^{-}\right), k=1,2, \ldots, b_{k} \geq 0$, and $\sum_{k=1}^{\infty} b_{k}<\infty$;

(iv) $0<u<v$ are given, $\phi^{(j)}(u)<v$; when $\tilde{h}_{0}^{(j)}\left(t, x_{t}^{(j)}\right)<u$, there holds $h^{(j)}(t) \leq$ $\phi^{(j)}\left(\tilde{h}_{0}^{(j)}\left(t, x_{t}^{(j)}\right)\right)$, where $\phi^{(j)}$ are wedge functions, and $x(t)=\left(x^{(1)}(t), x^{(2)}(t)\right)$ is a solution of (2.1) and (2.2).

Then the zero solution of $(2.1)$ and $(2.2)$ is $\left(\tilde{h}_{0}, h\right)$ uniformly practically stable with respect to $(u, v)$.

Proof. Since $b_{k} \geq 0$, and $\sum_{k=1}^{\infty} b_{k}<\infty$, it follows that there exists some $M>0$, such that $\prod_{k=1}^{\infty}\left(1+b_{k}\right)=M$ and $1 \leq M<\infty$. Define a function $V(t)$ for all $t \geq \alpha$

$$
V(t)=V_{1}(t) \quad \text { if } V_{1}(t) \geq V_{2}(t) ; \quad V(t)=V_{2}(t) \quad \text { if } V_{2}(t) \geq V_{1}(t)
$$


We claim first that for any $t \geq \alpha$

$$
\begin{aligned}
\frac{\left[W_{11}\left(h^{(1)}(t)\right)+W_{12}\left(h^{(2)}(t)\right)\right]}{2} \leq V(t) \leq & W_{21}\left(h_{0}^{(1)}(t)\right)+W_{22}\left(h_{0}^{(2)}(t)\right) \\
& +W_{31} \int_{\alpha}^{t} \Phi_{1}(t-s) W_{41}\left(h_{0}^{(1)}(t)\right) d s \\
& +W_{32} \int_{\alpha}^{t} \Phi_{2}(t-s) W_{42}\left(h_{0}^{(2)}(t)\right) d s
\end{aligned}
$$

In fact, if $V_{1}(t) \geq V_{2}(t)$, then by (3.1) and condition (i), $V(t)=V_{1}(t) \geq\left[V_{1}(t)+V_{2}(t)\right] / 2 \geq$ $\left[W_{11}\left(h^{(1)}(t)\right)+W_{12}\left(h^{(2)}(t)\right)\right] / 2$; whereas, if $V_{2}(t) \geq V_{1}(t)$, it also holds. On the other hand, the right-hand inequality in (3.2) is trivially valid.

Step 1 . we aim to show that for each $t \geq t_{0}$,

$$
\begin{gathered}
V^{\prime}(t) \leq 0, \quad \text { if } V(s) \leq V(t), \quad s \in[\alpha, t], t \neq \tau_{k}, \\
V\left(\tau_{k}\right) \leq\left(1+b_{k}\right) V\left(\tau_{k}^{-}\right), \quad k=1,2, \ldots
\end{gathered}
$$

Indeed, suppose $V_{1}\left(t_{0}\right) \geq V_{2}\left(t_{0}\right)$ and there exists some $t_{1}>t_{0}$ such that for $t \in\left[t_{0}, t_{1}\right], V_{1}(t) \geq$ $V_{2}(t)$. Then by (3.1), $V(t)=V_{1}(t), t \in\left[t_{0}, t_{1}\right]$.

Case 1. If $t=\tau_{j}$ for some $j \in Z^{+}$, then By (iii) $V\left(\tau_{j}\right)=V_{1}\left(\tau_{j}\right) \leq\left(1+b_{j}\right) V_{1}\left(\tau_{j}^{-}\right)=\left(1+b_{j}\right) V\left(\tau_{j}^{-}\right)$.

Case 2. $t \neq \tau_{j}$ for any $j \in Z^{+}$, and $V(s) \leq V(t), s \in[\alpha, t]$. Then if $V_{1}(s) \leq V_{2}(s)$ we have $V(s)=V_{2}(s)$. Clearly, $V(s) \leq V(t)$ implies $V_{1}(s) \leq V_{2}(s)=V(s) \leq V(t)=V_{1}(t)$. If $V_{1}(s) \geq$ $V_{2}(s)$ we have $V(s)=V_{1}(s)$. Obviously, $V(s) \leq V(t)$ implies $V_{1}(s)=V(s) \leq V(t)=V_{1}(t)$. In conclusion, $V(s) \leq V(t), s \in[\alpha, t], t \neq \tau_{k}$, implies $V_{1}(s) \leq V_{1}(t), s \in[\alpha, t], t \neq \tau_{k}$. So by (ii) we have $V^{\prime}(t)=V_{1}^{\prime}(t) \leq 0$.

If $t_{1}=\infty$ we arrive at the assertion that (3.3) is true for all $t \geq t_{0}$. Otherwise, there exists a $t_{2}>t_{1}$ such that $V_{1}(t) \leq V_{2}(t), t \in\left[t_{1}, t_{2}\right]$. When $t_{1}=\tau_{i}$ for some $i \in Z^{+}$we have $V_{1}\left(\tau_{i}^{-}\right) \geq V_{2}\left(\tau_{i}^{-}\right)$and $V_{1}\left(\tau_{i}\right) \leq V_{2}\left(\tau_{i}\right)$. In this case, by (iii) we have $V\left(\tau_{i}\right)=V_{2}\left(\tau_{i}\right) \leq(1+$ $\left.b_{i}\right) V_{2}\left(\tau_{i}^{-}\right) \leq\left(1+b_{i}\right) V\left(\tau_{i}^{-}\right)$. When $t_{1} \neq \tau_{i}$ for any $i \in Z^{+}$, we set $V(t)=V_{2}(t)$ for $t \in\left[t_{1}, t_{2}\right]$.

By the similar analysis to Cases 1 and 2, we also have (3.3) when $t, \tau_{k} \in\left[t_{1}, t_{2}\right]$.

If $t_{2}=\infty$ then (3.3) holds for all $t \geq t_{0}$. Otherwise, repeat the above argument to arrive at the assertion that (3.3) is valid for all $t \geq t_{0}$. As for the case of $V_{1}(t) \leq V_{2}(t)$ for $t \in\left[t_{0}, t_{1}\right]$, the process is similar and thus omitted.

For any $t_{0} \in R^{+}$, we assume there is a unique solution of (2.1), (2.2) through $\left(t_{0}, \varphi\right)$. Furthermore, we denote

$$
h(t, x(t)):=\max \left\{h^{(j)}(t), j=1,2\right\} ; \quad \tilde{h}_{0}(t):=\max \left\{\tilde{h}_{0}^{(j)}\left(t, x_{t}^{(j)}\right), j=1,2\right\}
$$


If $\left(t_{0}, x_{t_{0}}\right) \in R^{+} \times P C\left(\left[\alpha, t_{0}\right], R^{n}\right)$, such that $\tilde{h}_{0}\left(t_{0}, x_{t_{0}}\right)<u$. By condition (iv),

$$
h^{(j)}\left(t_{0}\right) \leq \phi^{(j)}\left(\tilde{h}_{0}^{(j)}\left(t_{0}\right)\right)<\phi^{(j)}(u)<v
$$

From the definition of $h(t, x(t))$, we have $h\left(t_{0}, x\left(t_{0}\right)\right)<v$.

Let $v^{*}=(1 / M) \min \left\{W_{11}(v), W_{12}(v)\right\}$, we assume $W_{2 j}(u)<v^{*} / 8$ and $W_{3 j}\left(J_{j} \times\right.$ $\left.W_{4 j}(u)\right)<v^{*} / 8$, where $J_{j}=\int_{0}^{\infty} \Phi_{j}(s) d s, j=1,2$.

Step 2. We aim to prove that $V(t) \leq M v^{*} / 2$, for all $t \geq t_{0}$.

First, for any $t \in\left[\alpha, t_{0}\right]$, from Definition 2.2 and condition (iv), we know $h_{0}^{(j)}\left(t, x^{(j)}(t)\right) \leq$ $\tilde{h}_{0}^{(j)}\left(t_{0}, x_{t_{0}}^{(j)}\right)<u$. Then by (3.2), $V_{j}(t) \leq W_{21}(u)+W_{22}(u)+W_{31}\left(J_{1} W_{41}(u)\right)+W_{32}\left(J_{2} W_{42}(u)\right)<$ $v^{*} / 2$ for $t \in\left[\alpha, t_{0}\right]$. Hence, $V(t) \leq v^{*} / 2, t \in\left[\alpha, t_{0}\right]$.

Assume $\tau_{l}$ is the first impulse of all $\tau_{i}, i \in Z^{+}$such that $t_{0}<\tau_{i}$. Now we claim that

$$
V(t) \leq \frac{v^{*}}{2} \text { for } t_{0} \leq t<\tau_{l}
$$

If it does not hold, then there is a $\widehat{t} \in\left(t_{0}, \tau_{l}\right)$ such that $V(\widehat{t})>v^{*} / 2$ and $V^{\prime}(\widehat{t})>0, V(t) \leq$ $V(\widehat{t})$ for $t \in[\alpha, \widehat{t}]$. From (3.3) we have $V^{\prime}(\widehat{t}) \leq 0$. It is a contradiction, so (3.6) holds.

Without loss of generality, we assume $V_{1}\left(\tau_{l}\right) \leq V_{2}\left(\tau_{l}\right)$, then $V\left(\tau_{l}\right)=V_{2}\left(\tau_{l}\right)$; from inequality (3.6) and condition (iii) we have $V\left(\tau_{l}\right)=V_{2}\left(\tau_{l}\right) \leq\left(1+b_{l}\right) V_{2}\left(\tau_{l}^{-}\right) \leq\left(1+b_{l}\right) v^{*} / 2$. Thus,

$$
V\left(\tau_{l}\right) \leq\left(1+b_{l}\right) \frac{v^{*}}{2}
$$

Similarly, with the process in proving (3.6) and (3.7), we have

$$
V(t) \leq\left(1+b_{l}\right) \frac{v^{*}}{2} \quad \text { for } \tau_{l} \leq t<\tau_{l+1} ; \quad V\left(\tau_{l+1}\right) \leq\left(1+b_{l+1}\right)\left(1+b_{l}\right) \frac{v^{*}}{2} .
$$

By simple induction, we can prove that, in general

$$
V(t) \leq\left(1+b_{l+i+1}\right) \cdots\left(1+b_{l}\right) \frac{v^{*}}{2} \quad \text { for } \tau_{l+i} \leq t \leq \tau_{l+i+1} .
$$

Taking this together with (3.2) and $\prod_{k=1}^{\infty}\left(1+b_{k}\right)=M$, we have

$$
\frac{\left[w_{11}\left(h^{(1)}(t)\right)+w_{12}\left(h^{(2)}(t)\right)\right]}{2} \leq V(t) \leq M \frac{v^{*}}{2}, \quad \forall t \geq t_{0}
$$

Since $M v^{*}=\min \left\{w_{11}(v), w_{12}(v)\right\}$, we have

$$
w_{1 j}\left(h^{(j)}(t)\right) \leq w_{1 j}(v), \quad \text { that is, } h^{(j)}(t) \leq v, j=1,2, \forall t \geq t_{0}
$$

Therefore, by the definition of $h(t, x)$, we have $h(t, x) \leq v$. Thus the zero solution of (2.1), (2.2) with respect to $(u, v)$ is $\left(\widetilde{h}_{0}, h\right)$-uniformly practically stable. 
Remark 3.2. Since in our result $\alpha$ may be $-\infty$ and the upper bound of the Lyapunov functions in our paper is improved by $w_{3 j}, j=1,2$, the result we have obtained is more general than that in $[4-7,14]$ with or without finite delay; furthermore, we have divided the components of $x$ into several groups, correspondingly, several Lyapunov functions $V_{j}\left(t, x^{(j)}\right)(j=1,2, \ldots, m)$ are employed, where $x=\left(x^{(1)}, \ldots, x^{(m)}\right)^{T}$ for each $x^{(j)}$. In this way, construction of the suitable Lyapunov functions is much easier than for $x$ as $[4,6,7,10]$. In additional, compared with $[9,12]$ where the infinite delay was considered in the Lyapunov stability of differential equations, we obtain the uniformly practical stability in terms of two measures.

Now, we may develop the ideas behind Theorem 3.1 to obtain the following more general results.

Theorem 3.3. For $j=1,2, \ldots, m$, let $\Phi_{j}: R^{+} \rightarrow R^{+}$be continuous, $\Phi_{j} \in L^{1}[0, \infty), \Phi_{j}(t) \leq K_{j}$ for $t \geq 0$ with some constants $K_{j}>0$, and let $W_{i j}(i=1,2,3,4)$ be wedge functions. If there also exist Lyapunov functions $V_{j}: R_{\alpha} \times S^{(j)}(H) \rightarrow R^{+}$such that

(i) $W_{1 j}\left(h^{(j)}(t)\right) \leq V_{j}(t) \leq W_{2 j}\left(h_{0}^{(j)}(t)\right)+W_{3 j}\left[\int_{\alpha}^{t} \Phi_{j}(t-s) W_{4 j}\left(h_{0}^{(j)}(t)\right) d s\right]$, where $h_{0}^{(j)} \in$ $\Gamma_{\alpha}^{n_{j}}, h^{(j)} \in \Gamma^{n_{j}}$;

(ii) when $V_{l}(t)=\max \left\{V_{j}(t) \mid j=1,2, \ldots, m\right\}$, there holds $V_{l}^{\prime}(t) \leq 0$ if $V_{l}(s)<V_{l}(t)$ for $s \in[\alpha, t] ; l=1,2, \ldots, m$;

(iii) $V_{j}\left(\tau_{k}\right) \leq\left(1+b_{k}\right) V_{j}\left(\tau_{k}^{-}\right), k=1,2, \ldots, b_{k} \geq 0$, and $\sum_{k=1}^{\infty} b_{k}<\infty$;

(iv) $0<u<v$ are given, $\phi^{(j)}(u)<v$; when $\tilde{h}_{0}^{(j)}\left(t, x_{t}^{(j)}\right)<u, h^{(j)}(t) \leq \phi^{(j)}\left(\tilde{h}_{0}^{(j)}\left(t, x_{t}^{(j)}\right)\right)$ where $\phi^{(j)}$ are wedge functions, and $x(t)=\left(x^{(1)}(t), \ldots, x^{(m)}(t)\right)$ is a solution of $(2.1)$ and (2.2).

Then the zero solution of $(2.1)$ and $(2.2)$ is $\left(\tilde{h}_{0}, h\right)$-uniformly practically stable.

It suffices to mention a few points in the proofs of Theorem 3.3, the rest are the same as in the proofing of Theorem 3.1, thus, are omitted.

First, for $x(t)=\left(x^{(1)}(t), \ldots, x^{(m)}(t)\right)$, we define

$$
V(t)=V_{l}(t), \quad V_{l}(t)=\max \left\{V_{j}(t) \mid j=1,2, \ldots, m\right\} ;
$$

Second, instead of (3.2) we can claim that for any $t \geq \alpha$

$$
\frac{\sum_{j=1}^{m} W_{1 j}\left(h^{(j)}(t)\right)}{m} \leq V(t) \leq \sum_{j=1}^{m} W_{2 j}\left(h_{0}^{(j)}(t)\right)+\sum_{j=1}^{m} W_{3 j} \int_{\alpha}^{t} \Phi_{j}(t-s) W_{4 j}\left(h_{0}^{(j)}(t)\right) d s .
$$

Example 3.4. Consider the equation

$$
\begin{gathered}
x_{1}^{\prime}(t)=-a_{1}(t) x_{1}(t)+a_{2}(t) x_{2}(t)+b_{1}(t) x_{1}\left(t-r_{1}(t)\right)+\int_{-\infty}^{0} g_{1}\left(t, u, x_{1}(t+u)\right) d u, \quad t \neq t_{k}, \\
x_{2}^{\prime}(t)=c_{1}(t) x_{1}(t)-c_{2}(t) x_{2}(t)+b_{2}(t) x_{2}\left(t-r_{2}(t)\right)+\int_{-\infty}^{0} g_{2}\left(t, u, x_{2}(t+u)\right) d u, \quad t \neq t_{k}, \\
x_{i}\left(t_{k}\right)-x_{i}\left(t_{k}^{-}\right)=I_{k}\left(x_{i}\left(t_{k}^{-}\right)\right), \quad k \in Z^{+}, i=1,2,
\end{gathered}
$$


where $\left|x+I_{k}(x)\right|^{2} \leq\left(1+b_{k}\right)^{2} x^{2}$, with $b_{k} \geq 0, \sum_{k=1}^{\infty} b_{k}<\infty$. Let $M=\prod_{k=1}^{\infty}\left(1+b_{k}\right)<\infty$. $a_{i}, b_{i}, c_{i}, r_{i}$ and $g_{i}(i=1,2)$ are all continuous functions.

We first assume that $r_{i}(t) \geq 0$ and $\left|g_{i}(t, u, x)\right| \leq m_{i}(u)|x|, t \geq 0, i=1,2$, with $\int_{-\infty}^{0} m_{1}(u) d u \leq a_{1}(t)-\left|a_{2}(t)\right|-\left|b_{1}(t)\right|$, and $\int_{-\infty}^{0} m_{2}(u) d u \leq c_{2}(t)-\left|c_{1}(t)\right|-\left|b_{2}(t)\right|$. Without loss of generality, we may assume that the right-hand sides of (3.14) are defined on $R \times P C_{1}(t)$, then set $\alpha=-\infty$ and $t_{*}=0$.

Let $V_{j}\left(t, x_{j}(t)\right)=x_{j}^{2}(t), h_{0}^{(j)}\left(t, x_{j}\right)=x_{j}^{2}(t), w_{1 j}(s)=(1 / 2) s, w_{2 j}(s)=2 s$, then from the definition $\tilde{h}_{0}^{(j)}\left(t, x_{j_{t}}\right)=\sup _{-\infty<\theta \leq t} x_{j}^{2}(\theta)=\left\|x_{j_{t}}^{2}\right\|, j=1$, 2 . For given $0<u<v$, we assume $\left\|x_{j_{t}}^{2}\right\|<u$ implies that there exists a $K \in R^{+}$such that $x_{j}^{2}(t)<K x_{j}^{2}(\theta)$ for any $\theta \in(-\infty, t]$. Let $h^{(j)}\left(t, x_{j}\right)=x_{j}^{2}(t) /(K+1), \phi^{(i)}(t)=(K /(K+1)) t$, then $\phi^{(i)}(u)<u<v$; furthermore, when $\tilde{h}_{0}^{(j)}\left(t, x_{j_{t}}\right)=\left\|x_{j_{t}}^{2}\right\|<u$, then for $\theta \in(-\infty, t]$,

$$
h^{(j)}\left(t, x_{j}\right)=\frac{x_{j}^{2}(t)}{K+1} \leq \frac{K}{K+1} x_{j}^{2}(\theta) \leq \frac{K}{K+1}\left\|x_{j_{t}}^{2}\right\|=\phi^{(j)}\left(\widetilde{h}_{0}^{(j)}\left(t, x_{j_{t}}\right)\right),
$$

so conditions (i) and (iv) in Theorem 3.1 are verified.

Moreover, when $V_{1}(t) \geq V_{2}(t)$, that is, $\left|x_{1}(t)\right| \geq\left|x_{2}(t)\right|$, and for $s \in(-\infty, t], V_{1}(s) \leq$ $V_{1}(t)$, we have

$$
\begin{aligned}
V_{1}^{\prime}(t)= & -2 a_{1}(t) x_{1}^{2}(t)+2 a_{2}(t) x_{1}(t) x_{2}(t)+2 b_{1}(t) x_{1}(t) x_{1}\left(t-r_{1}(t)\right) \\
& +2 x_{1}(t) \int_{-\infty}^{0} g_{1}\left(t, u, x_{1}(t+u)\right) d u \\
\leq & -2\left[a_{1}(t)-\left|a_{2}(t)\right|-\left|b_{1}(t)\right|-\int_{-\infty}^{0} m_{1}(u) d u\right] x_{1}^{2}(t) \leq 0,
\end{aligned}
$$

similarly, when $V_{1}(t) \leq V_{2}(t)$ and for $s \in(-\infty, t], V_{2}(s) \leq V_{2}(t)$, we also have $V_{2}^{\prime}(t) \leq 0$. Thus, condition (ii) in Theorem 3.1 is satisfied and the zero solution of system $(3.14)$ is $\left(\tilde{h}_{0}, h\right)$ uniformly practically stable.

It is easy to see that if we put two variables $x_{1}, x_{2}$ in one Lyapunov function, then the arguments to get the desired stability conclusions would be much more complicated and the imposed conditions would be more restrictive. Furthermore, we extend the uniformly practically stable results to the infinite delay systems, and it is easy to see that the criteria in [3-10] are limited to judge the practical stability of Example 3.4.

\section{Acknowledgments}

This work was supported by the National Natural Science Foundation of China (nos. 11101373 and 61074011), the Natural Science Foundation of Zhejiang Province of China (no. Y6100007), and Zhejiang Innovation Project (no. T200905). 


\section{References}

[1] J. LaSalle and S. Lefschetz, Stability by Liapunov's Direct Method, with Applications, Academic Press, New York, NY, USA, 1961.

[2] A. A. Martynyuk, Practical Stability of Motion, Naukova Dumka, Kiev, Russia, 1983.

[3] V. Lakshmikantham, S. Leela, and A. A. Martynyuk, Practical Stability of Nonlinear Systems, World Scientific, Singapore, 1990.

[4] Y. Zhang and J. Sun, "Practical stability of impulsive functional differential equations in terms of two measurements," Computers \& Mathematics with Applications, vol. 48, no. 10-11, pp. 1549-1556, 2004.

[5] Y. Liu and S. Zhao, "A new approach to practical stability of impulsive functional differential equations in terms of two measures," Journal of Computational and Applied Mathematics, vol. 223, no. 1, pp. 449-458, 2009.

[6] I. M. Stamova, "Vector Lyapunov functions for practical stability of nonlinear impulsive functional differential equations," Journal of Mathematical Analysis and Applications, vol. 325, no. 1, pp. 612-623, 2007.

[7] Y. Zhang and J. Sun, "Eventual practical stability of impulsive differential equations with time delay in terms of two measurements," Journal of Computational and Applied Mathematics, vol. 176, no. 1, pp. 223-229, 2005.

[8] S. M. S. de Godoy and M. A. Bená, "Stability criteria in terms of two measures for functional differential equations," Applied Mathematics Letters, vol. 18, no. 6, pp. 701-706, 2005.

[9] X. Li, "Uniform asymptotic stability and global stabiliy of impulsive infinite delay differential equations," Nonlinear Analysis: Theory, Methods E Applications, vol. 70, no. 5, pp. 1975-1983, 2009.

[10] X. Liu and Q. Wang, "On stability in terms of two measures for impulsive systems of functional differential equations," Journal of Mathematical Analysis and Applications, vol. 326, no. 1, pp. 252-265, 2007.

[11] Y. Liu, S. Zhao, and J. Lu, "A new fuzzy impulsive control of chaotic systems based on T-S fuzzy model," IEEE Transactions on Fuzzy Systems, vol. 19, no. 2, pp. 393-398, 2011.

[12] Z. Luo and J. Shen, "Stability of impulsive functional differential equations via the Liapunov functional," Applied Mathematics Letters, vol. 22, no. 2, pp. 163-169, 2009.

[13] J. H. Shen, "Razumikhin techniques in impulsive functional-differential equations," Nonlinear Analysis: Theory, Methods \& Applications, vol. 36, no. 1, pp. 119-130, 1999.

[14] I. M. Stamova, "Lyapunov-Razumikhin method for impulsive differential equations with 'supremum'," IMA Journal of Applied Mathematics, vol. 76, no. 4, pp. 573-581, 2011.

[15] B. Wu, Y. Liu, and J. Q. Lu, "New results on global exponential stability for impulsive cellular neural networks with any bounded time-varying delays," Mathematical and Computer Modelling, vol. 55, pp. 837-843, 2012.

[16] B. Wu, Y. Liu, and J. Q. Lu, "Impulsive control of chaotic systems and its applications in synchronization," Chinese Physics, vol. 20, no. 5, Article ID 050508, 2011.

[17] M. De La Sen and N. Luo, "A note on the stability of linear time-delay systems with impulsive inputs," IEEE Transactions on Circuits and Systems. I. Fundamental Theory and Applications, vol. 50, no. 1, pp. 149$152,2003$.

[18] M. De la Sen, "Stability of impulsive time-varying systems and compactness of the operators mapping the input space into the state and output spaces," Journal of Mathematical Analysis and Applications, vol. 321, no. 2, pp. 621-650, 2006.

[19] D. Chen, G. Yang, and Z. Han, "Impulsive observer for input-to-state stability based synchronization of Lur'e differential inclusion system," Communications in Nonlinear Science and Numerical Simulation, vol. 17, pp. 2990-2996, 2012.

[20] Y. Xing and M. Han, "A new approach to stability of impulsive functional differential equations," Applied Mathematics and Computation, vol. 151, no. 3, pp. 835-847, 2004.

[21] S. Zhang, "A new approach to stability theory of functional-differential equations," Annals of Differential Equations, vol. 11, no. 4, pp. 495-503, 1995.

[22] T. Yang, Impulsive Systems and Control, Theory and Applications, Nova Science, Huntington, NY, USA, 2001. 


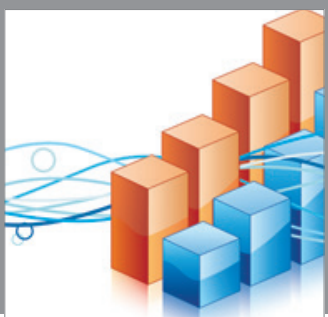

Advances in

Operations Research

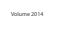

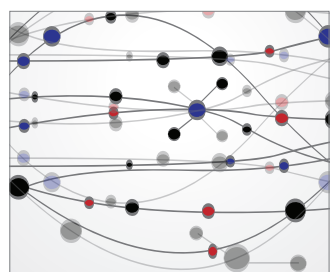

\section{The Scientific} World Journal
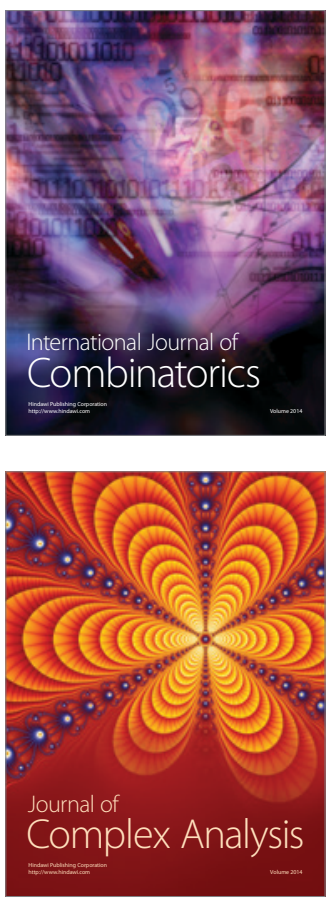

International Journal of

Mathematics and

Mathematical

Sciences
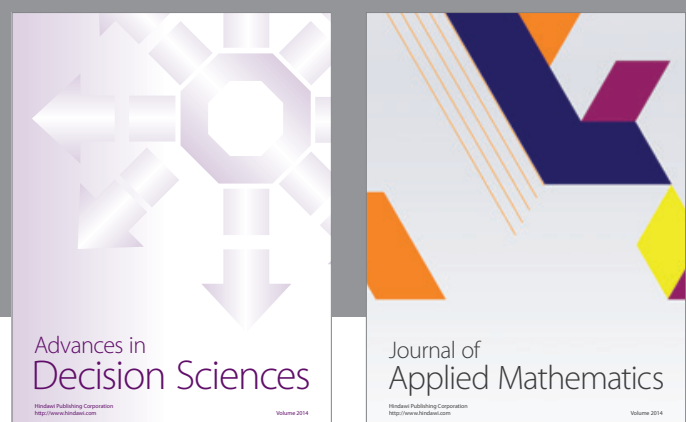

Journal of

Applied Mathematics
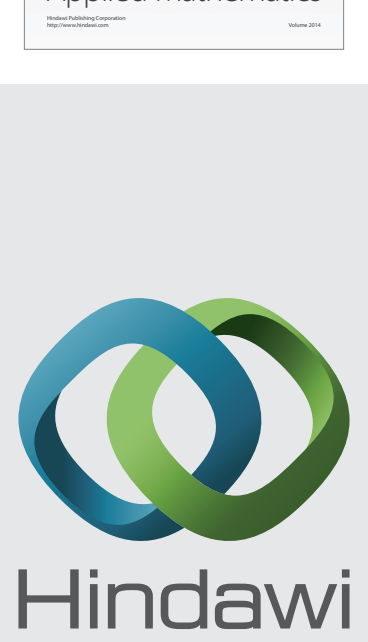

Submit your manuscripts at http://www.hindawi.com
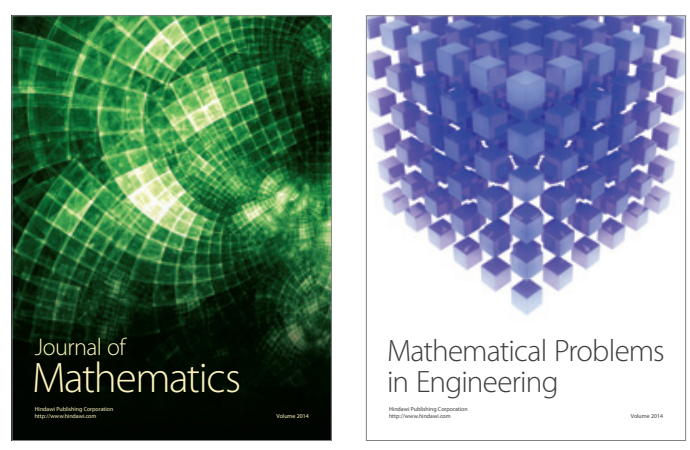

Mathematical Problems in Engineering
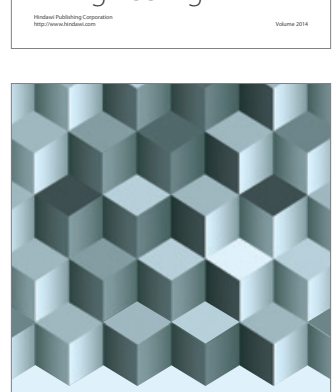

Journal of

Function Spaces
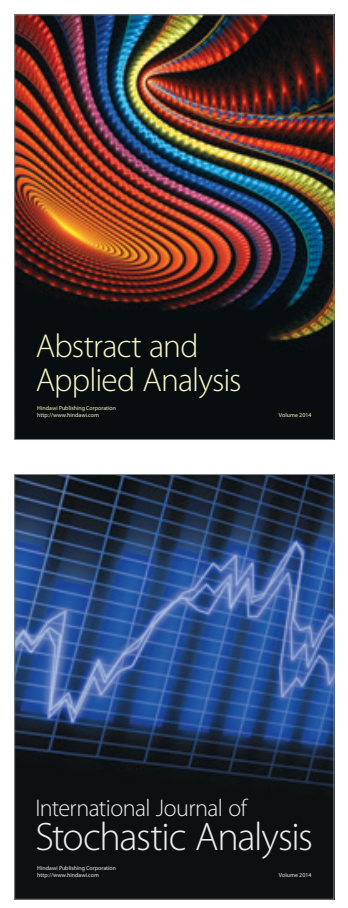

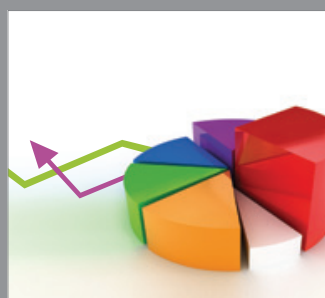

ournal of

Probability and Statistics

Promensencen
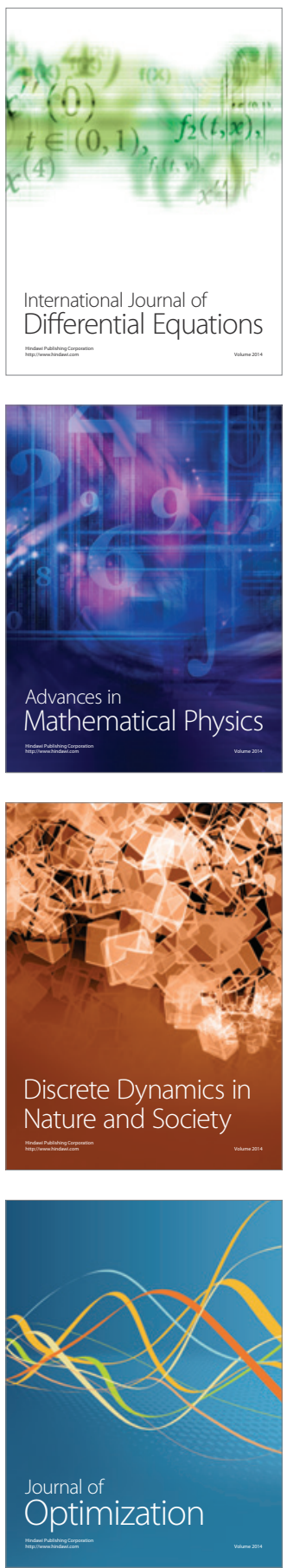